\title{
Telomere protein RAP1 levels are affected by cellular aging and oxidative stress
}

\author{
MARK J. SWANSON ${ }^{1,2}$, MICHELLE E. BARIBAULT ${ }^{2}$, JOANNA N. ISRAEL ${ }^{2}$ and NANCY S. BAE ${ }^{2}$ \\ ${ }^{1}$ Division of Basic Medical Sciences, Mercer University School of Medicine, Macon, GA 30207; ${ }^{2}$ Department of \\ Biochemistry, Arizona College of Osteopathic Medicine, Midwestern University, Glendale, AZ 85308, USA
}

Received January 22, 2016; Accepted June 24, 2016

DOI: $10.3892 /$ br.2016.707

\begin{abstract}
Telomeres are important for maintaining the integrity of the genome through the action of the shelterin complex. Previous studies indicted that the length of the telomere did not have an effect on the amount of the shelterin subunits; however, those experiments were performed using immortalized cells with stable telomere lengths. The interest of the present study was to observe how decreasing telomere lengths over successive generations would affect the shelterin subunits. As neonatal human dermal fibroblasts aged and their telomeres became shorter, the levels of the telomere-binding protein telomeric repeat factor 2 (TRF2) decreased significantly. By contrast, the levels of one of its binding partners, repressor/activator protein 1 (RAP1), decreased to a lesser extent than would be expected from the decrease in TRF2. Other subunits, TERF1-interacting nuclear factor 2 and protection of telomeres protein 1, remained stable. The decrease in RAP1 in the older cells occurred in the nuclear and cytoplasmic fractions. Hydrogen peroxide $\left(\mathrm{H}_{2} \mathrm{O}_{2}\right)$ stress was used as an artificial means of aging in the cells, and this resulted in RAP1 levels decreasing, but the effect was only observed in the nuclear portion. Similar results were obtained using U251 glioblastoma cells treated with $\mathrm{H}_{2} \mathrm{O}_{2}$ or grown in serum-depleted medium. The present findings indicate that TRF2 and RAP1 levels decrease as fibroblasts naturally age. RAP1 remains more stable compared to TRF2. RAP1 also responds to oxidative stress, but the response is different to that observed in aging.
\end{abstract}

Correspondence to: Dr Nancy S. Bae, Department of Biochemistry, Arizona College of Osteopathic Medicine, Midwestern University, 19555 N 59th Avenue, Glendale, AZ 85308, USA

E-mail:nbae@midwestern.edu

Abbreviations: HDFn, human dermal fibroblasts derived from neonatal foreskin; PDL, population doubling level

Key words: telomere, aging, shelterin, repressor/activator protein 1, telomeric repeat factor 2, human dermal fibroblasts, oxidative stress

\section{Introduction}

Telomeres are the specialized nucleoprotein structures found at the ends of the linear chromosomes of eukaryotic cells. They are comprised of tandem arrays of short DNA sequence repeats. Human telomeres are composed of several kilobases of 5'-TTAGGG-3' sequences on what is referred to as the G-rich strand. The majority of the telomere repeats are found as duplexes, but the 3 ' end of the G-rich strand extends as a single strand. Telomeres do not encode proteins. Instead, their repeat sequences partly serve as a 'buffer' that can be lost when the 5 ' terminal RNA primers cannot be replaced following DNA replication [reviewed in (1)]. The telomere length is normally maintained in embryonic cells and stem cells by the activity of telomerase. However, telomeres are shortened with each successive round of DNA replication in cells that no longer express telomerase, such as terminally differentiated skin fibroblasts. Generally, once telomeres reach a critically short length, cells become senescent (2).

Shelterin is a six-member protein complex that associates with telomeric DNA in mammals [reviewed in (3)]. In cells with telomerase activity, shelterin has a role in maintaining telomere length. This complex is generally required to protect the ends of the chromosomes from proteolytic degradation and prevent them from being recognized as double-stranded DNA breaks. Without the protection of shelterin, DNA repair mechanisms would be triggered, fusing the chromosomes end-to-end resulting in genomic instability (3).

The six subunits of shelterin are telomeric repeat factor 1 (TRF1), TRF2, TERF1-interacting nuclear factor 2 (TIN2), protection of telomeres protein 1 (POT1), tripeptidyl peptidase 1 (TPP1) and repressor/activator protein 1 (RAP1). Among the subunits, TRF1 and TRF2 bind to the duplex region of the repeat array $(4,5)$. Although structurally similar (6), TRF1 and TRF2 have extremely different roles at the telomeres and bind to distinct sets of partner proteins [reviewed in (7)]. TRF1 negatively regulates telomere length (8) and facilitates telomere DNA replication (9), while TRF2 is essential for telomere capping (3) and protecting the telomeres from DNA damage repair mechanisms (10). TRF1 and TRF2 interact with TIN2. TIN2 stabilizes the TRF1-TRF2 interaction onto telomeric DNA (6). TRF2 also binds to RAP1, but TRF1 does not (11).

The POT1 and TPP1 subunits of the complex are interacting partners $(12,13)$, and the two proteins are represented 
in equal amounts in cells (14). The POT1 protein is found at the 3 ' end of the G-rich strand, bound to the single-stranded TTAGGG repeats (15). TPP1 is required for the stability of POT1 (16). The TPP1-POT1 complex prevents a DNA damage repair response at the telomeric overhang site $(17,18)$. TPP1 is also involved in connecting POT1 to TIN2 $(13,19,20)$. As TIN2 also binds to TRF1 and TRF2, it serves to connect all of the DNA binding activities of shelterin (21-23).

Of the six shelterin subunits, the highly conserved RAP1 protein is the only subunit that is not essential in mice $(24,25)$. As part of shelterin, RAP1 contributes to the maintenance of genome stability by protecting telomeric DNA ends from non-homologous end joining $(26,27)$ and from homologous recombination that can alter telomere length (24). RAP1 is recruited to telomeric repeats by TRF2, and in mouse cells, RAP1 levels are dependent on TRF2 (10). In humans, RAP1 is more abundant than TRF2, and knocking down TRF2 resulted in a reduction in RAP1 (14). By contrast, immunodepletion of RAP1 resulted in a loss of TRF2 from cell extracts $(26,27)$.

RAP1 has non-telomeric activities as well. In the nucleus, mouse RAP1 associates with non-telomeric chromatin, and a knockout leads to an alteration in the expression of a set of genes, about a third of which contained TTAGGG sequences in their promoter regions (25). In human cells, RAP1 binds to selective telomeric sequences located at interstitial sites and regulate gene transcription (28). In addition to its nuclear functions, RAP1 has been found in the cytoplasm of human cells. RAP1 is required for inhibitor of nuclear factor (NF)- $\kappa B$ kinase (IKK) phosphorylation of the p65 subunit of $N F-\kappa B$, making p65 competent for transcriptional activation (29).

The telomere is a dynamic structure, and the interaction between its proteins and DNA is key to the integrity of the genome. Progressive shortening of telomeres that occurs in normal, somatic cells suggests that telomere stability is disrupted over time since there should be fewer available binding sites for shelterin to bind. A previous study assessed whether there was a correlation between various lengths of telomere sequences and the levels of the shelterin components (14). However, the study was performed using immortalized cells with varying length telomeres that maintained those lengths generation after generation. A quantitative analysis of shelterin components in cells that undergo normal telomere attrition would be informative in understanding the natural aging process. In the present study, the progression of normal, somatic cells was followed through their natural life span using neonatal human dermal fibroblasts.

Telomere lengths and cellular phenotypes of young cells (low population doubling) and old cells (high population doubling) were observed and the levels of shelterin components in the two populations were compared. In addition, the expression levels of RAP1 were evaluated in the nucleus and the cytoplasm of cells that were naturally or prematurely aged by hydrogen peroxide $\left(\mathrm{H}_{2} \mathrm{O}_{2}\right)$-induced oxidative stress or via serum deprivation.

\section{Materials and methods}

Cell lines. Human dermal fibroblasts derived from neonatal foreskin (HDFn) and growth medium were obtained from Invitrogen (Thermo Fisher Scientific, Inc., Waltham, MA,
USA). HDFn cells were grown at $37^{\circ} \mathrm{C}$ with $5 \% \mathrm{CO}_{2}$ in medium 106 supplemented with low serum growth supplement. Cells were passaged according to the manufacturer's protocol. The population doubling levels (PDLs) of cells were calculated as follows: PDL $=3.32 \times\left(\log \mathrm{X}_{\text {harvest }}-\log \mathrm{X}_{\text {seeding }}\right)+$ starting PDL, where $X_{\text {harvest }}$ is the number of cells at the time of harvest and $\mathrm{X}_{\text {seeding }}$ is the number of cells seeded (30). The U251 glioblastoma cell line was kindly provided by Dr Prakash Chinnaiyan from the H. Lee Moffitt Cancer Center and Research Institute (Tampa, FL, USA). U251 cells were propagated in Dulbecco's modified Eagle's medium (DMEM) supplemented with $10 \%$ fetal bovine serum (FBS) in $5 \% \mathrm{CO}_{2}$ environment at $37^{\circ} \mathrm{C}$. At each passage, the cells were grown to $80-100 \%$ confluence and were subsequently split at a 1:5 ratio.

Telomere length analysis. To isolate genomic DNA, HDFn cells were grown in $100-\mathrm{mm}$ plates. When the cells were $\sim 95 \%$ confluent, the genomic DNA from a desired PDL was isolated using the DNeasy ${ }^{\circledR}$ Blood and Tissue kit (Qiagen, Valencia, CA, USA). The lengths of telomeres were analyzed using the TeloTAGGG Telomere Length assay from Roche Life Sciences US (Indianapolis, IN, USA) according to the manufacturer's protocol. Briefly, equal amounts $(1.5 \mu \mathrm{g})$ of genomic DNA from young and old cells were digested with RsaI and HinfI, which digest non-telomeric sequences but leave telomere intact. The generated DNA fragments were resolved on a $0.8 \%$ agarose gel for $4 \mathrm{~h}$ at $80 \mathrm{~V}$. Transfer of the DNA fragments onto a nylon membrane by capillary action occurred for $24 \mathrm{~h}$. DNA was cross-linked with UV to the nylon membrane using a Stratalinker (Stratagene, Santa Clara, CA, USA) according to the manufacturer's protocol. Both prehybridization and hybridization were performed at $42^{\circ} \mathrm{C}$ overnight with gentle agitation. Detection of the signal was performed using autoradiography. Calculation of the mean telomere lengths was determined using Telometric (31).

Cell extract preparation and fractionation. For whole cell extracts, HDFn cells were grown in 100-mm dishes to $\sim 95 \%$ confluence. At the desired population doubling, cells were washed with cold $1 \mathrm{X}$ phosphate-buffered saline (PBS) and lysed in radioimmunoprecipitation assay buffer $[150 \mathrm{mM}$ $\mathrm{NaCl}, 1.0 \% \mathrm{NP}-40,0.5 \%$ deoxycholate, $0.1 \%$ SDS and $50 \mathrm{mM}$ Tris- $\mathrm{HCl}$ ( $\mathrm{pH}$ 8.0)] containing protease inhibitors (cOmplete EDTA-free Protease Inhibitor Cocktail Tablet; Sigma-Aldrich, St. Louis, MO, USA) for $2 \mathrm{~min}$. The cells were scraped from the surface of the flasks, transferred to microcentrifuge tubes and centrifuged at $20,000 \mathrm{x}$ g for $5 \mathrm{~min}$. The soluble fractions were transferred to new tubes. For cellular fractionation, the cells were washed with cold $1 \mathrm{X}$ PBS and fractionated as previously described (32). Protein concentrations of all samples were determined using the Pierce ${ }^{\circledR}$ BCA Protein assay kit (Thermo Fisher Scientific, Inc., Chicago, IL, USA).

Induction of oxidative stress. Oxidative damage was introduced to HDFn cells by treatment with $\mathrm{H}_{2} \mathrm{O}_{2}$ as follows. The cells were grown in $100-\mathrm{mm}$ dishes to $\sim 95 \%$ confluence to a PDL of 9. Short-term oxidative stress was induced by incubating cells in medium containing a final concentration of $20 \mu \mathrm{M}$ $\mathrm{H}_{2} \mathrm{O}_{2}$. The cells were exposed to the $\mathrm{H}_{2} \mathrm{O}_{2}$ for $2 \mathrm{~h}$ at $37^{\circ} \mathrm{C}$. Cells without $\mathrm{H}_{2} \mathrm{O}_{2}$ treatment were run in parallel as a control. 
After the incubation, treated and untreated cells were washed with $1 \mathrm{X}$ PBS and fresh growth medium was added. Four days post-treatment, images of the cells were captured, and the were subsequently fractionated as previously described (32).

U251 cells were stressed using $\mathrm{H}_{2} \mathrm{O}_{2}$ or serum deprivation. The cells were grown in 100-mm dishes to $~ 80 \%$ confluence in DMEM/10\% FBS. Short-term oxidative stress was induced by adding $\mathrm{H}_{2} \mathrm{O}_{2}$ at a final concentration of $1 \mathrm{mM}$ for $3 \mathrm{~h}$ at $37^{\circ} \mathrm{C}$. For serum-starvation, cells were grown in DMEM/0.5\% FBS at $37^{\circ} \mathrm{C}$ overnight. Cells without $\mathrm{H}_{2} \mathrm{O}_{2}$ treatment, grown in $\mathrm{DMEM} / 10 \% \mathrm{FBS}$ were run in parallel as a control. Following the incubation, cells were washed with $1 \mathrm{X}$ PBS and harvested, lysed and fractionated as previously described (32).

Immunoblotting. The samples were resolved on $10 \%$ SDS-polyacrylamide gels and were transferred onto nitrocellulose membranes. Blocking and incubation with antibodies was performed in 5\% non-fat dry milk/10 mM Tris (pH 7.5), $150 \mathrm{mM} \mathrm{NaCl}$ and $0.05 \%$ Tween-20 (TBST). Membranes were washed with TBST. The primary antibodies used in the study were obtained from the following vendors: mouse monoclonal anti-human TRF2 (cat. no. 05-521) from Calbiochem (Billerica, MA, USA) used at 1:1,000 dilution; rabbit polyclonal anti-human RAP1 (cat. no. A300-306A) from Bethyl Laboratories (Montgomery, TX, USA) used at 1:2,000 dilution; rabbit polyclonal anti-human TIN2 (cat. no. ab82998) from Abcam Inc. (Cambridge, MA, USA) used at 1:1,000 dilution; rabbit polyclonal anti-human POT1 (cat. no. NB500-176) from Novus Biologicals (Littleton, CO, USA) used at 1:1,000 dilution; and goat polyclonal anti-human actin (cat. no. sc-1616) from Santa Cruz Biotechnology, Inc. (Santa Cruz, CA, USA) used at 1:2,000 dilution. Secondary antibodies conjugated to horseradish peroxidase were used in conjunction with the SuperSignal West Pico chemiluminescent kit (Thermo Fisher Scientific, Inc.) for detection of the proteins in Figs. 1B, 2 and 3. For Fig. 1A, protein detection was performed using the Odyssey (LI-COR Biosciences, Lincoln, NE, USA) with the secondary antibodies IRDye 800CW (cat. no. 925-32211) and IRDye 680RD (cat. no. 925-68070) used at 1:50,000 dilutions. The intensities of the bands were determined either densitometrically using ImageJ (33) or using the Odyssey CLx imager quantitation program.

\section{Results}

HDFn cells at increased PDLs exhibit hallmarks of aging. The present study aimed to determine the fate of the shelterin subunits in normal, differentiated human cells with a finite number of divisions as they age using HDFn cells. The cells grew for specific numbers of PDL and young cells of only a few PDL were compared with old cells isolated following numerous doublings. Before analyzing the shelterin complex, cell aging was confirmed by examining the cellular morphologies and telomere lengths of young and old cells. HDFn cells isolated at 3 or 6 PDL were considered to be young, and cells at PDL28, PDL32 and PDL38 were considered old. These PDLs were chosen to represent old cells to avoid using senescent cells that have telomeres that have become critically short and are no longer dynamically changing. At PDL6, the cells appeared
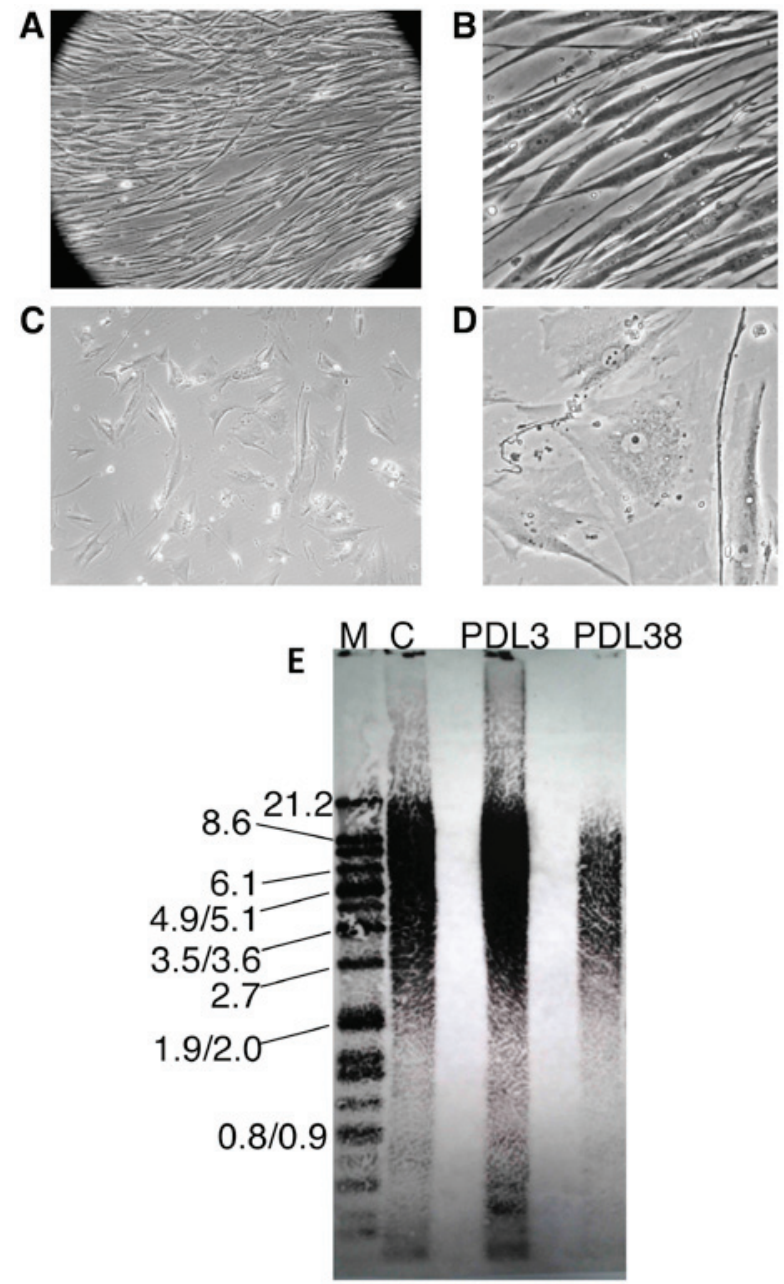

Figure 1. Effects of aging in HDFn cells. Morphologies of HDFn cells were visualized microscopically at different population doublings. Visible light microscopic images were taken of young HDFn cells at PDL6 (A,B) and old HDFn cells at PDL38 (C,D). The photographs were taken at 100x magnification $(\mathrm{A}, \mathrm{C})$ and $\mathrm{x} 200(\mathrm{~B}, \mathrm{D})$. (E) Telomere length analysis was performed on young and old HDFn cells. Lane M contains the molecular weight ladder in kilobase pairs (kbp), and the sizes in kbp of several of the marker bands are listed to the left of the image. Lane $\mathrm{C}$ contains the telomere smear of control DNA. The lane labeled PDL3 contains the telomere smear of young HDFn cells at population doubling 3. The lane labeled PDL38 contains the telomere smear of aged HDFn cells at population doubling 38. Smears represent varying telomere lengths in a heterogeneous population of cells. Mean telomere length was determined using the Telometric software, and they are $10.3 \mathrm{kbp}$ for the control DNA, 10.6 kbp for PDL3 and 5.1 kbp for PDL38.

long and slender with spindle-like features characteristic of young fibroblasts (Fig. 4A and B). By contrast, cells at PDL38 were irregularly shaped, more flattened with the presence of large vacuoles and had an increased cytoplasm volume (Fig. 4C and D). As HDFn cells are terminally differentiated, their telomeres are expected to shorten with each round of DNA replication. The telomere lengths were measured in young (PDL3) and old (PDL38) HDFn cells. As expected, the telomere lengths were heterogeneous (Fig. 4E). The mean terminal restriction fragment length for the HDFn cells at PDL3 was $\sim 10.5$ kilobase pair (kbp) while it was $\sim 5.1 \mathrm{kbp}$ for the HDFn cells at PDL38. In addition to the decreased length of the telomeres, the telomeric signal of the DNA isolated from the old cells was significantly reduced compared to the younger cells, indicating less telomeric DNA in the older population. 
A
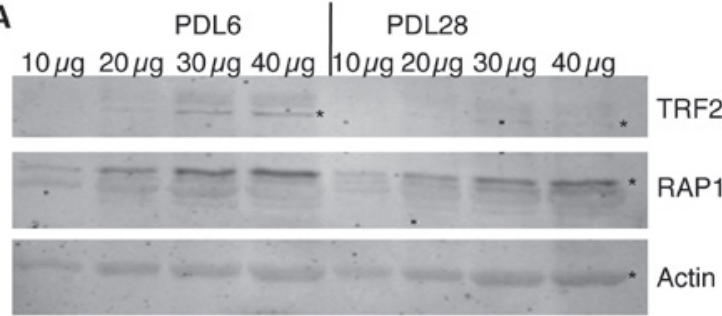

B

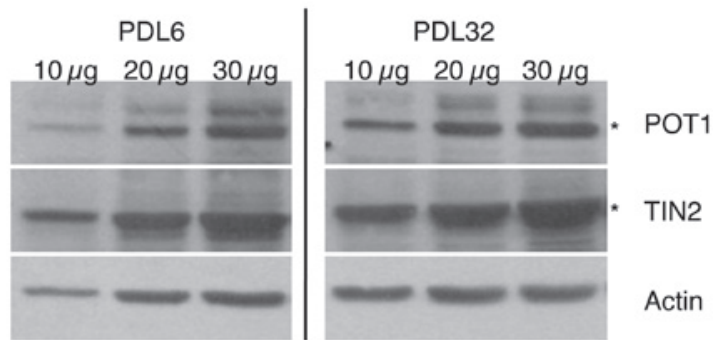

Figure 2. Shelterin levels in young and old HDFn cells. (A) The levels of TRF2 (upper panel) and RAP1 (middle panel) were compared in young HDFn cells at PDL6 and old HDFn cells at PDL28. Various amounts of whole cell extracts were resolved by SDS-PAGE, and the blotted proteins were detected with specific antibodies. Actin was used as a loading control (lower panel). The secondary antibodies used were for detection using the Odyssey CLx imager quantitation program (LI-COR Biosciences). Asterisks indicate the appropriate bands for the proteins of interest. Measurements were done using the Odyssey CLx imager quantitation program. Values for TRF2 and RAP1 were normalized by those for actin. The values calculated for each lane of one protein were averaged for each PDL. TRF2 levels in the PDL28 samples were $36 \%$ of the PDL6 levels. RAP1 at PDL28 was $75 \%$ of the PDL6 levels. (B) The levels of POT1 (upper panel) and TIN2 (middle panel) were compared in young HDFn cells at PDL6 and old HDFn cells at PDL32. Various amounts of whole cell extracts were resolved by SDS-PAGE, and the blotted proteins were detected with specific antibodies. Actin was used as a loading control (lower panel). Asterisks indicate the appropriate bands for the proteins of interest. Measurements for the appropriate bands on the film were done using NIH ImageJ. Values for POT1 and TIN2 were normalized by those for actin. The values calculated for each lane of one protein were averaged for each PDL. Levels of both POT1 and TIN2 in the older cells were approximately the same as those in the younger cells. For both A and B, all portions of the figure were from the same blot with irrelevant information deleted for visual clarity.

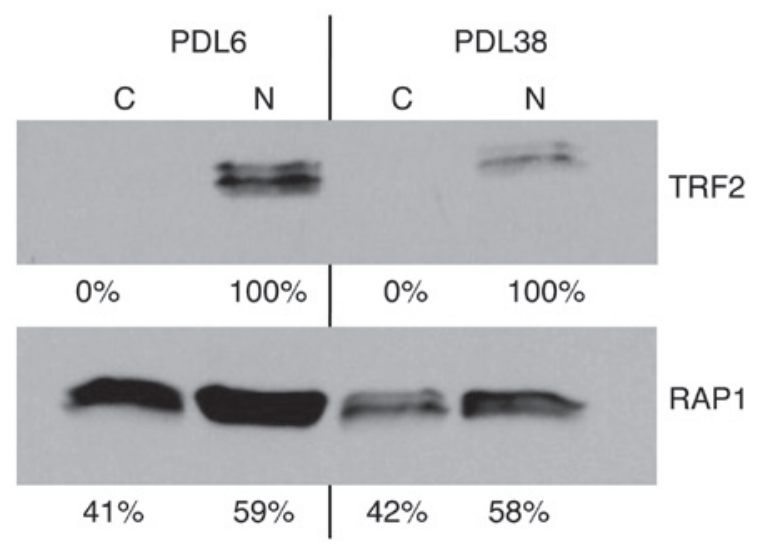

Figure 3. Cellular distribution of RAP1 and TRF2 in young and old HDFn cells. Cell lysates from young (PDL6) and old (PDL38) were fractionated into cytoplasmic (labeled $\mathrm{C}$ ) and nuclear (labeled N) portions before resolving proteins by SDS-PAGE and immunoblotting. Equal amounts of protein were loaded in each lane. The levels of TRF2 (upper panel) and RAP1 (lower panel) were measured using NIH ImageJ. The numbers under the panels indicate the proportion of the total signal (cytoplasmic and nuclear) that was found in the specific fraction. The total amount of RAP1 in the older cells was $53 \%$ that of the younger cells. The total amount of TRF2 in the older cells was $26 \%$ the level of the young cells.

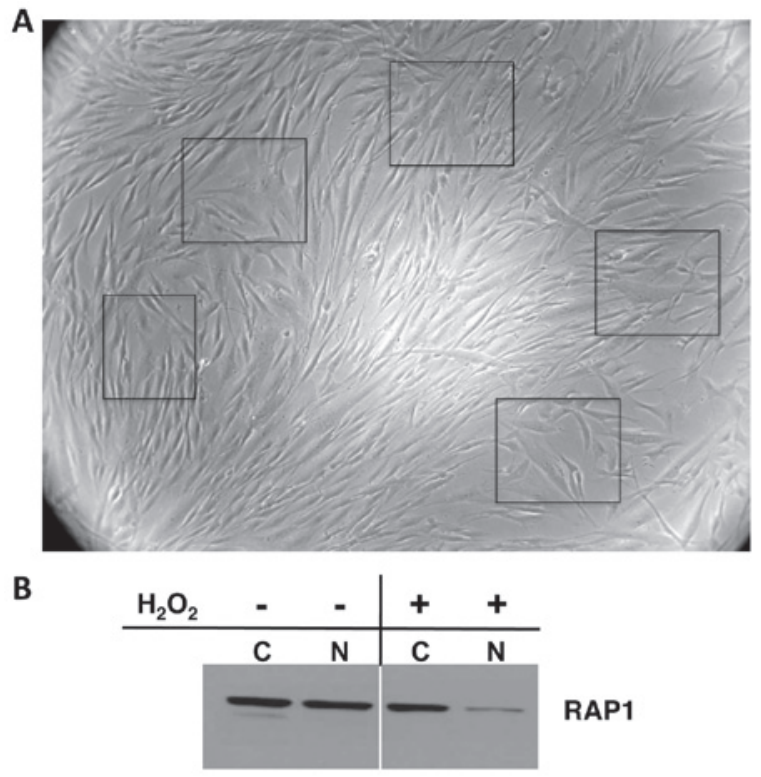

C

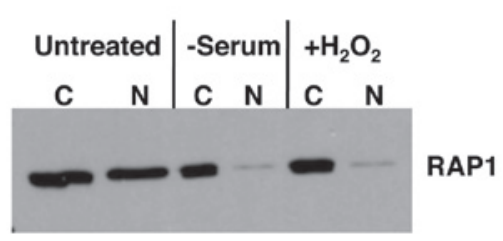

Figure 4. Oxidative stress affects the distribution of RAP1 in HDFn and U251 cells. (A) HDFn cells at PDL9 were treated with $20 \mu \mathrm{M} \mathrm{H}_{2} \mathrm{O}_{2}$ for $2 \mathrm{~h}$. Cells were photographed at 100x magnification. The black boxes on the micrograph indicate cells that show the most obvious aged-cell morphologies. (B) Lysates of HDFn cells at PDL9 with or without $\mathrm{H}_{2} \mathrm{O}_{2}$ treatment were fractionated into cytoplasmic (C) and nuclear (N) portions as in A. Proteins were resolved by SDS-PAGE, and immunoblotting was performed to detect RAP1. Images were from the same blot with irrelevant information deleted for visual clarity. (C) U251 cells were grown in normal medium (Untreated), serum-depleted medium overnight (-Serum) or medium with $1 \mathrm{mM} \mathrm{H}_{2} \mathrm{O}_{2}$ for $3 \mathrm{~h}\left(+\mathrm{H}_{2} \mathrm{O}_{2}\right)$. Cell lysates were fractionated into cytoplasmic $(\mathrm{C})$ and nuclear (N) portions. Proteins were resolved by SDS-PAGE, and immunoblotting was performed to detect RAP1.

Abundance of shelterin components as telomeres shorten in aging cell populations. The levels of various shelterin subunits in young and old HDFn cells were measured. When comparing TRF2 levels of older (PDL28) and younger (PDL6) cells, the amounts of TRF2 were diminished in older cells to approximately one-third of those observed in the younger cells (Fig. 1A). However, when RAP1, which requires TRF2 to direct it to the telomeres, was measured from the same samples, the level in the older cells was $75 \%$ that of the younger cells.

The POT1 subunit binds to the single-stranded repeats at the end of the G-rich strand. The TIN2 protein forms a bridge between POT1 and the rest of the shelterin complex. Levels of the single-stranded DNA binding protein POT1 and its interacting partner TIN2 were compared between young (PDL6) and old (PDL32) cell populations. The POT1 and TIN2 proteins appeared to be at approximately the same levels in the young and old cells (Fig. 2B).

Subcellular localization of RAP1 and TRF2 in young versus old cell populations. To determine where in the cells the RAP1 and TRF2 proteins were located, nuclear and cytoplasmic fractions of young (PDL3) and old (PDL38) HDFn 
cell lysates were analyzed. As expected, TRF2 was only found in the nucleus (Fig. 2, upper panel). RAP1 was found in the nuclear and cytoplasmic fractions (Fig. 2, lower panel). When measuring total levels of TRF2 and RAP1 by adding the amounts for each fraction together and subsequently comparing old and young cells, the TRF2 and RAP1 levels at PDL38 (Fig. 2) had continued to decline as the cells aged from PDL28 (Fig. 1A). In the older (PDL38) cells, TRF2 levels were $26 \%$ of those from the PDL6 population, while RAP1 levels of PDL11 were $53 \%$ of those of PDL6. Although decreased, the levels of RAP1 continued to remain higher in the older population. However, the decrease in RAP1 levels was distributed between the nuclear and cytoplasmic pools.

Effects of oxidative stress on RAP1. When young HDFn cells were treated with $\mathrm{H}_{2} \mathrm{O}_{2}$ they showed typical aged-cell morphologies (Fig. 3A compared to cells at PDL38 in Fig. 4C and D).

To determine the effects of oxidative stress on RAP1, HDFn cells were treated with $\mathrm{H}_{2} \mathrm{O}_{2}$ and fractionated into cytoplasmic and nuclear portions. In the untreated cells, RAP1 levels in the cytoplasm and nucleus were similar, but $\mathrm{H}_{2} \mathrm{O}_{2}$ treatment resulted in a loss of the nuclear RAP1 with no effect on the cytoplasmic pool (Fig. 3B). To determine whether this phenomenon was a general feature of all cells or just those without telomerase activity as in the HDFn cells, $\mathrm{U} 251$ glioblastoma cells were exposed to $\mathrm{H}_{2} \mathrm{O}_{2}$. Similar to the results of the $\mathrm{H}_{2} \mathrm{O}_{2}$-treated HDFn cells, the nuclear RAP1 was severely depleted (Fig. 3C). Withdrawing serum, which results in oxidative stress, from U251 cells led to RAP1 levels in the cytoplasm and nucleus that were similar to the levels in cells treated with $\mathrm{H}_{2} \mathrm{O}_{2}$ (Fig. $3 \mathrm{C}$ ).

\section{Discussion}

Telomeres protect the ends of linear chromosomes, providing genomic stability. The DNA repeats of the telomere serve as a binding platform for the shelterin complex. As opposed to the rest of the genome in normal, terminally differentiated cells, the telomere is a dynamic structure. As cells replicate their DNA for cell division, the telomere shortens due to an inability to replace the terminal RNA primers. Thus, the length of the telomere limits the number of replication cycles in cells without telomerase, the enzyme that normally extends telomeres in embryonic and stem cells.

As cells progress through numerous divisions, telomere shortening is believed to result in a reduced number of binding sites for the shelterin complex, leading to the expectation that shelterin subunit levels may decrease concomitantly. A previous study showed that the levels of the shelterin subunits did not reflect telomere lengths (14). However, those experiments were performed using immortalized cell lines with telomeres that were stable across generations. To the best of our knowledge, the present study is the first to compare levels of shelterin components between younger and older populations in a single-cell lineage of human dermal fibroblasts as they age.

HDFn with a finite lifespan were grown and isolated at early passages (young cells) or after numerous PDLs (old cells). Particular PDLs for the old cells were chosen, such that the cells exhibited aging cell morphologies and shortened telomeres (Fig. 4) without being completely senescent, in order to determine the fate of shelterin as the telomere is changing. As telomere repeats decrease in number over generations, the single-stranded DNA overhangs at the ends of the telomere should remain stable. Congruently, the levels of POT1, which bind to the single-stranded DNA of the telomere, were the same in young and old cells (Fig. 1B). TPP1 is one of the binding partners of POT1 $(12,13)$, and TPP1 and POT1 are found in a 1:1 stoichiometry in shelterin (14), thus the levels of TPP1 were not assessed. TIN2 connects POT1/TPP1 to the remainder of shelterin (21-23). TIN2 levels in the present study also did not change as cells aged (Fig. 1B).

As the telomere lengths decrease, the levels of TRF2 decreased (Fig. 1A). This result would support the general hypothesis that the loss of potential binding sites in the telomere would affect the abundance of telomeric protein. Technical difficulties complicated the measurement of TRF1. However, previous study has shown that TRF1 levels are substoichiometric compared to the levels of TRF2 (14), so we would speculate that the levels of TRF1 would also decrease at a rate similar to that of TRF2. The levels of RAP1, the binding partner of TRF2, also decreased in older cells, but the decrease was less than that of TRF2 (Fig. 1A). These data are in line with RAP1 being involved in one or more non-telomeric functions, possibly DNA binding for the control for the control of gene expression in the nucleus $(25,28)$ and as an effector protein for NF- $\mathrm{B}$ signaling in the cytoplasm (29) as cells age.

Since RAP1 has been found to play roles in both the nucleus and cytoplasm, we undertook experiments to determine where the decreases in RAP1 levels occurred in older cells. As has been shown previously $(29,14)$, we only detected TRF2 in the nucleus of cells, but RAP1 was found in both the nuclear and cytoplasmic fractions (Fig. 2). Interestingly, the decrease in the levels of RAP1 was distributed between the nucleus and the cytoplasm with the levels in each compartment decreasing the same relative amount. The decrease in the amount of RAP1 in the nucleus most likely reflects the decrease in TRF2. The RAP1 remaining in the nucleus is probably made up of both RAP1 that is associated with the telomere repeats that remain and the RAP1 bound at other chromosomal locations. It was surprising that the cytoplasmic levels of RAP1 also declined in the older cells. Since RAP1 has been shown to be a positive regulator of $\mathrm{NF}-\kappa \mathrm{B}$ signaling (29), some RAP1 may remain in the cytoplasm to carry out that function. NF- $\kappa \mathrm{B}$ normally provides cells with protection from apoptosis (34 and references therein). Based on the finding that knocking down RAP1 sensitized breast tumor cells to apoptosis (29), it is possible that RAP1 in the cytoplasm may decrease over successive generations to allow aged cells to become more susceptible to apoptosis as well. This may provide a mechanism by which cells that are old and more likely damaged (e.g., through the accumulation of ROS and DNA damage) will undergo apoptosis rather than become immortalized. Another possibility is that the levels of RAP1 may decrease in older cells so that a different effector protein can regulate $\mathrm{NF}-\kappa \mathrm{B}$ signaling. Since increased $\mathrm{NF}-\kappa \mathrm{B}$ activation makes fibroblasts less susceptible to reprogramming (35), it seems likely that RAP1 may be exchanged for some other signaling factor. This may allow the cells to alter 
their responses to various stimuli between young and old cells. Hypothetically, decreased RAP1 levels may help provide a barrier to cellular reprogramming and sensitize cells to apoptosis. Additional study in this area seems prudent.

One factor known to affect aging is oxidative stress. As cells replicate, oxidative damage accumulates, and this can be seen in cellular phenotypes. We treated our young neonatal human dermal fibroblasts with $\mathrm{H}_{2} \mathrm{O}_{2}$ to cause premature aging. The levels of $\mathrm{H}_{2} \mathrm{O}_{2}$ we used resulted in cellular morphologies reminiscent of old cells (Fig. 3A), which correlate with previous studies, though telomeres did not shorten (36). Using these conditions, nuclear RAP1 levels were greatly diminished while the levels in the cytoplasm remained relatively stable (Fig. 3B). Similar results were obtained when U251 glioblastoma cells were treated with $\mathrm{H}_{2} \mathrm{O}_{2}$, indicating that the decrease in RAP1 levels occurs in cells that express telomerase, maintaining long telomeres, as well as in the HDFn cells that do not express telomerase. Serum depletion has been shown to increase the occurrence of ROS in cells. We depleted serum from the growth medium of U251 cells, the RAP1 levels in the nucleus decreased, but those in the cytoplasm remained stable, similarly to the $\mathrm{H}_{2} \mathrm{O}_{2}$ treatment (Fig. 3C). Overall, RAP1 levels in the nucleus decreased when the cells were treated with $\mathrm{H}_{2} \mathrm{O}_{2}$ or serum-depleted medium, suggesting a possible role for RAP1 in the response to oxidative stress. The maintenance of RAP1 in the cytoplasm of the stressed, young HDFn cells and the immortalized glioblastoma cells seems likely due to maintaining $\mathrm{NF}-\kappa \mathrm{B}$ signaling to promote cell survival. The conditions used for the $\mathrm{H}_{2} \mathrm{O}_{2}$ treatment of the HDFn cells do not result in a loss of telomeric repeats (36), and yet there is a loss of nuclear RAP1. One possible explanation is that the cytoplasmic RAP1 is turned over rapidly during $\mathrm{NF}-\kappa \mathrm{B}$ signaling under such extreme conditions, and the nuclear RAP1 translocates to maintain the cytoplasmic pool for continued signaling.

\section{Acknowledgements}

The present study was supported by Midwestern University College of Health Sciences and intramural funding from Midwestern University.

\section{References}

1. Campisi J: Aging, cellular senescence, and cancer. Annu Rev Physiol 75: 685-705, 2013.

2. Blackburn EH: Telomere states and cell fates. Nature 408: 53-56, 2000.

3. de Lange T: Shelterin: The protein complex that shapes and safeguards human telomeres. Genes Dev 19: 2100-2110, 2005.

4. Bianchi A, Smith S, Chong L, Elias P and de Lange T: TRF1 is a dimer and bends telomeric DNA. EMBO J 16: 1785-1794, 1997.

5. Broccoli D, Smogorzewska A, Chong L and de Lange T: Human telomeres contain two distinct Myb-related proteins, TRF1 and TRF2. Nat Genet 17: 231-235, 1997.

6. Court R, Chapman L, Fairall L and Rhodes D: How the human telomeric proteins TRF1 and TRF2 recognize telomeric DNA: A view from high-resolution crystal structures. EMBO Rep 6: 39-45, 2005.

7. Palm W and de Lange T: How shelterin protects mammalian telomeres. Annu Rev Genet 42: 301-334, 2008.

8. Ancelin K, Brunori M, Bauwens S, Koering CE, Brun C, Ricoul M, Pommier JP, Sabatier L and Gilson E: Targeting assay to study the cis functions of human telomeric proteins: Evidence for inhibition of telomerase by TRF1 and for activation of telomere degradation by TRF2. Mol Cell Biol 22: 3474-3487, 2002 .
9. Sfeir A, Kosiyatrakul ST, Hockemeyer D, MacRae SL, Karlseder J, Schildkraut CL and de Lange T: Mammalian telomeres resemble fragile sites and require TRF1 for efficient replication. Cell 138: 90-103, 2009.

10. Celli GB and de Lange T: DNA processing is not required for ATM-mediated telomere damage response after TRF2 deletion. Nat Cell Biol 7: 712-718, 2005.

11. Li B, Oestreich S and de Lange T: Identification of human Rap1: Implications for telomere evolution. Cell 101: 471-483, 2000.

12. Xin H, Liu D, Wan M, Safari A, Kim H, Sun W, O'Connor MS and Songyang Z: TPP1 is a homologue of ciliate TEBP-beta and interacts with POT1 to recruit telomerase. Nature 445: 559-562, 2007.

13. Liu D, Safari A, O'Connor MS, Chan DW, Laegeler A, Qin J and Songyang Z: PTOP interacts with POT1 and regulates its localization to telomeres. Nat Cell Biol 6: 673-680, 2004.

14. Takai KK, Hooper S, Blackwood S, Gandhi R and de Lange T: In vivo stoichiometry of shelterin components. J Biol Chem 285: 1457-1467, 2010

15. Baumann P and Cech TR: Pot1, the putative telomere end-binding protein in fission yeast and humans. Science 292: 1171-1175, 2001.

16. Hockemeyer D, Palm W, Else T, Daniels JP, Takai KK, Ye JZ, Keegan CE, de Lange T and Hammer GD: Telomere protection by mammalian Pot1 requires interaction with Tpp1. Nat Struct Mol Biol 14: 754-761, 2007.

17. Churikov D and Price CM: Pot1 and cell cycle progression cooperate in telomere length regulation. Nat Struct Mol Biol 15: 79-84, 2008.

18. Guo X, Deng Y, Lin Y, Cosme-Blanco W, Chan S, He H, Yuan G, Brown EJ and Chang S: Dysfunctional telomeres activate an ATM-ATR-dependent DNA damage response to suppress tumorigenesis. EMBO J 26: 4709-4719, 2007

19. Wang F, Podell ER, Zaug AJ, Yang Y, Baciu P, Cech TR and Lei M: The POT1-TPP1 telomere complex is a telomerase processivity factor. Nature 445: 506-510, 2007.

20. Houghtaling BR, Cuttonaro L, Chang W and Smith S: A dynamic molecular link between the telomere length regulator TRF1 and the chromosome end protector TRF2. Curr Biol 14: 1621-1631, 2004.

21. Ye JZ, Donigian JR, van Overbeek M, Loayza D, Luo Y, Krutchinsky AN, Chait BT and de Lange T: TIN2 binds TRF1 and TRF2 simultaneously and stabilizes the TRF2 complex on telomeres. J Biol Chem 279: 47264-47271, 2004.

22. Kim SH, Beausejour C, Davalos AR, Kaminker P, Heo SJ and Campisi J: TIN2 mediates functions of TRF2 at human telomeres. J Biol Chem 279: 43799-43804, 2004.

23. Chen LY, Liu D and Songyang Z: Telomere maintenance through spatial control of telomeric proteins. Mol Cell Biol 27: 5898-5909, 2007.

24. Sfeir A, Kabir S, van Overbeek M, Celli GB and de Lange T: Loss of Rap1 induces telomere recombination in the absence of NHEJ or a DNA damage signal. Science 327: 1657-1661, 2010.

25. Martinez P, Thanasoula M, Carlos AR, Gómez-López G, Tejera AM, Schoeftner S, Dominguez O, Pisano DG, Tarsounas M and Blasco MA: Mammalian Rap1 controls telomere function and gene expression through binding to telomeric and extratelomeric sites. Nat Cell Biol 12: 768-780, 2010.

26. Bae NS and Baumann P: A RAP1/TRF2 complex inhibits nonhomologous end-joining at human telomeric DNA ends. Mol Cell 26: 323-334, 2007.

27. Sarthy J, Bae NS, Scrafford J and Baumann P: Human RAP1 inhibits non-homologous end joining at telomeres. EMBO J 28: 3390-3399, 2009.

28. Yang D, Xiong Y, Kim H, He Q, Li Y, Chen R and Songyang Z: Human telomeric proteins occupy selective interstitial sites. Cell Res 21: 1013-1027, 2011.

29. Teo H, Ghosh S, Luesch H, Ghosh A, Wong ET, Malik N, Orth A, de Jesus P, Perry AS, Oliver JD, et al: Telomere-independent Rap1 is an IKK adaptor and regulates NF-kappaB-dependent gene expression. Nat Cell Biol 12: 758-767, 2010.

30. Hayflick L: Subculturing human diploid fibroblast cultures. In: Tissue Culture Methods and Applications. Kruse PF Jr and Patterson MK (eds). Academic Press, New York, NY, pp220-223, 1973.

31. Grant JD, Broccoli D, Muquit M, Manion FJ, Tisdall J and Ochs MF: Telometric: A tool providing simplified, reproducible measurements of telomeric DNA from constant field agarose gels. Biotechniques 31: 1314-1316, 1318, 2001. 
32. Méndez J and Stillman B: Chromatin association of human origin recognition complex, cdc6, and minichromosome maintenance proteins during the cell cycle: Assembly of prereplication complexes in late mitosis. Mol Cell Biol 20: 8602-8612, 2000 .

33. Abramoff MD, Magalhaes PJ and Ram SJ: Image Processing with ImageJ. Biophoton Int 11: 36-42, 2004.

34. Xia Y, Shen S and Verma IM: NF- $\kappa$ B, an active player in human cancers. Cancer Immunol Res 2: 823-830, 2014.
35. Soria-Valles C, Osorio FG, Gutiérrez-Fernández A, De Los Angeles A, Bueno C, Menéndez P, Martín-Subero JI, Daley GQ, Freije JM and López-Otín C: NF- $\kappa B$ activation impairs somatic cell reprogramming in ageing. Nat Cell Biol 17: 1004-1013, 2015.

36. Chen QM, Prowse KR, Tu VC, Purdom S and Linskens MH: Uncoupling the senescent phenotype from telomere shortening in hydrogen peroxide-treated fibroblasts. Exp Cell Res 265: 294-303, 2001. 